\title{
Una especie nueva de Vaejovis (Scorpiones: Vaejovidae) del centro de México
}

\section{A new species of Vaejovis (Scorpiones: Vaejovidae) from central Mexico}

\author{
Erwin Pabel Miranda-López ${ }^{1}$, Javier Ponce-Saavedra ${ }^{1 \otimes}$ y Oscar F. Francke ${ }^{2}$ \\ ${ }^{1}$ Laboratorio de Entomología Biólogo Sócrates Cisneros Paz, Facultad de Biología, Universidad Michoacana de San Nicolás de Hidalgo. Edificio B-4 \\ 2 o piso, Ciudad Universitaria 58060 Morelia, Michoacán, México. \\ ${ }^{2}$ Colección Nacional de Arácnidos, Departamento de Zoología, Instituto de Biología, Universidad Nacional Autónoma de México. Apartado postal \\ 70-153, 04510 México, D. F., México. \\ 凶javierpon@gmail.com
}

\begin{abstract}
Resumen. Se describe Vaejovis morelia sp. nov., de Morelia, Michoacán, México. La especie se ubica en el grupo mexicanus (sensu Sissom, 2000) y es morfológicamente similar a $V$. pusillus Pocock, 1898 y V. dzahui Santibañez y Francke 2010, especies de zonas montañosas y con las que se compara.

Palabras clave: sistemática, Vaejovis, complejo pusillus, métodos morfométricos.

Abstract. Vaejovis morelia sp. nov. from Morelia, Michoacán, Mexico is described. This species belongs to the mexicanus group (sensu Sissom, 2000), and is morphologically similar to V. pusillus Pocock, 1898 and V. dzahui Santibañez y Francke 2010, species from mountainous zones, and with which it is compared.
\end{abstract}

Key words: systematics, Vaejovis, pusillus complex, morphometric methods.

\section{Introducción}

Por su amplia diversidad específica, la familia Vaejovidae y en particular el género Vaejovis Koch, 1836 han sido objeto de numerosos estudios y revisiones de los que se han derivado propuestas para su clasificación e incluso controversias al respecto (Sissom, 2000; Prendini et al., 2005; Soleglad y Fet, 2008). A la fecha se conocen 70 especies de Vaejovis que se distribuyen desde el suroeste de los Estados Unidos hasta Guatemala, siendo el territorio mexicano donde se encuentra su mayor diversidad (Sissom, 2000; Santibáñez-López y Sissom, 2010). De acuerdo con la clasificación propuesta por Sissom (2000) el género Vaejovis está subdividido en 5 grupos: eusthenura, intrepidus, mexicanus, nitidulus y punctipalpi; además de un grupo de especies que se ubica como incertae sedis.

El grupo mexicanus es heterogéneo y en su mayoría lo componen especies pequeñas. Se distribuye desde el sur de Estados Unidos hasta el sur del México y cuenta aproximadamente con 34 especies (Sissom, 2000; Hendrixson y Sissom, 2001; Graham, 2007; Zárate-Gálvez y Francke, 2009; Graham y Bryson, 2010; SantibáñezLópez y Francke, 2010; Ayrey y Soleglad, 2011; Sissom, 2011). Varias de estas especies son morfológicamente muy parecidas, con hábitats similares en tipo de vegetación (bosques de pino y de pino-encino) y altitud (localidades

Recibido: 26 marzo 2012; aceptado: 26 junio 2012 conocidas, ubicadas entre los 1800 y los $2400 \mathrm{~m}$ ) y poblaciones distribuidas en parches o islas. Este es el caso de las diversas poblaciones que hay en México, particularmente en Michoacán y estados vecinos, como Guanajuato, Guerrero y Estado de México, que en la actualidad se reconocen como Vaejovis pusillus Pocock, 1898 (Miranda et al., 2010).

El estado de Michoacán cuenta con una amplia área geográfica con altitudes superiores a los $1800 \mathrm{~m}$, debido a la presencia de 2 sierras importantes: la sierra Madre del Sur y el Eje Neovolcánico Transversal (Atlas geográfico del Estado de Michoacán, 2003), quedando varias montañas aisladas con potencial para el estudio de la escorpiofauna con distribución en parches. El presente trabajo es resultado de un estudio sobre este tipo de poblaciones y presenta la descripción de una nueva especie del grupo mexicanus del estado de Michoacán.

\section{Materiales y métodos}

Se revisaron 10 ejemplares adultos (5 machos y 5 hembras) provenientes del bosque Lázaro Cárdenas, municipio de Morelia, Michoacán, ubicado en los 1940.76' $\mathrm{N}, 101^{\circ} 10.65^{\prime} \mathrm{O}$ y a una altitud de $2030 \mathrm{~m}$ (Fig. 1). Los individuos fueron colectados en la noche entre la hojarasca y troncos caídos utilizando lámparas con luz negra cercana al U.V., aprovechando la propiedad de fluorescencia de la cutícula al contacto con esta luz (Stahnke, 1972). Se 


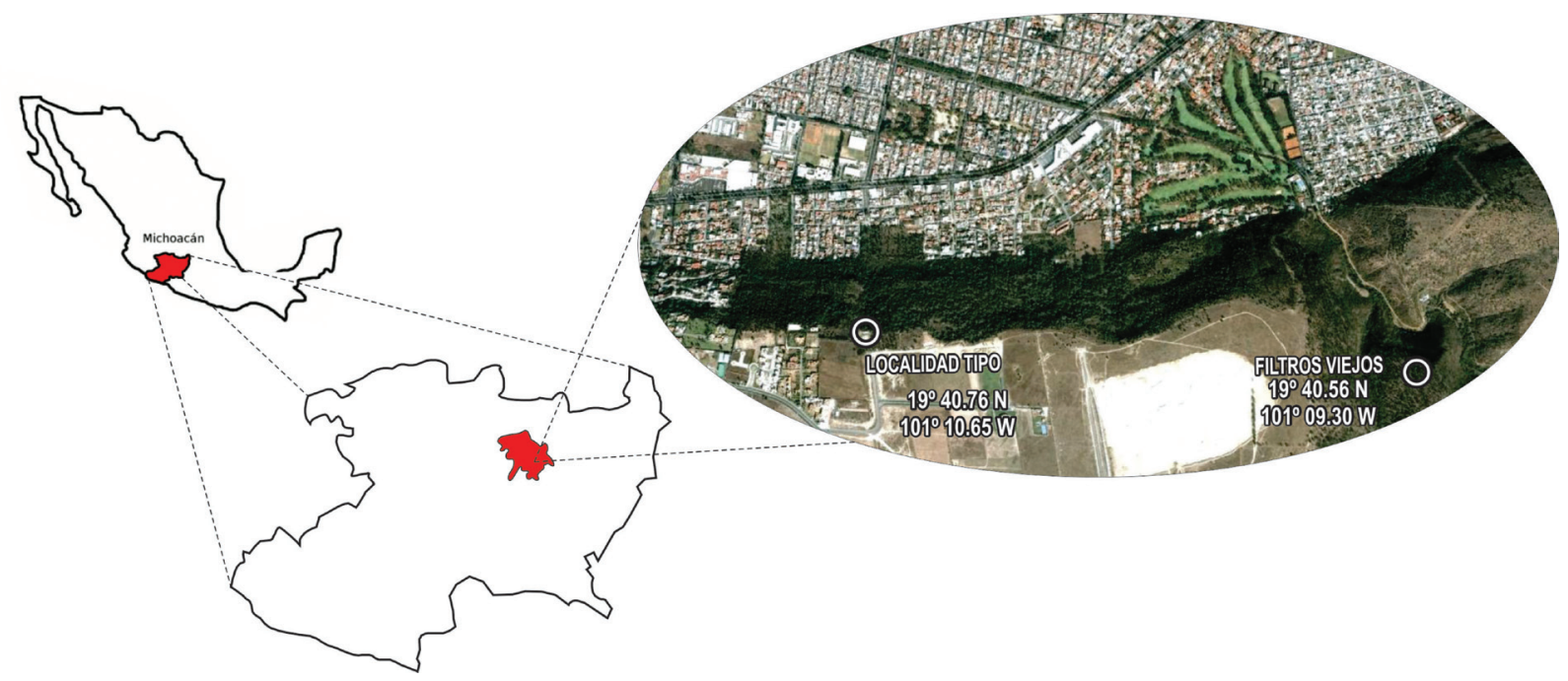

Figura 1. Ubicación de la localidad tipo y la localidad de Los Filtros Viejos en el municipio de Morelia, Michoacán. Ampliación modificada de Google Earth ${ }^{\circledR}$.

sacrificaron por choque térmico y se conservaron en alcohol etílico al 80\% (Sissom et al., 1990).

Todos los ejemplares se revisaron con un microscopio estereoscópico Zeiss Stemi DV4 y se midieron con un micrómetro con precisión de $0.1 \mathrm{~mm}$. Se hizo el conteo de dientes pectíneos y se tomaron 23 medidas (Cuadro 1). Las mediciones se realizaron de acuerdo con Sissom et al. (1990). Las comparaciones estadísticas entre hembras y machos se hicieron con una prueba no paramétrica para 2 muestras. En la descripción y nomenclatura se utilizó la terminología propuesta por Stahnke (1970); en la tricobotriotaxia, la de Vachon (1974); en las carinas metasomales, la sugerida por Francke (1977) y para el pedipalpo, la propuesta por Acosta et al. (2008). Para tener una mejor apreciación del patrón de granulación los ejemplares se revisaron secos de acuerdo con lo indicado por Santibáñez-López y Sissom (2010). El conteo de las setas de los esternitos se realizó con base en lo sugerido por Santibañez-López y Francke (2010). Para las setas metasomales se siguió la propuesta de Santibáñez-López y Sissom (2010).

Se hizo la comparación directa con 5 machos y 4 hembras de Vaejovis pusillus de Omiltemi, Guerrero $\left(17^{\circ} 33.27^{\prime} \mathrm{N}, 99^{\circ} 21.77^{\prime} \mathrm{O}\right)$, localidad tipo de la especie y con 4 machos y 4 hembras de Vaejovis dzahui Santibañez-López y Francke, 2010, especie cercana a $V$. pusillus, de San Cristóbal Suchixtlahuaca-Santiago Tejupam, Oaxaca $\left(17^{\circ} 42.240^{\prime} \mathrm{N}, 9^{\circ} 23.667^{\prime} \mathrm{O}\right)$. Todos los ejemplares provienen de la Colección Nacional de Arácnidos (CNAN) del Instituto de Biología de la UNAM (IBUNAM).

\section{Descripción}

Vaejovis morelia sp. nov.

Diagnosis. Vaejovis morelia sp. nov. se ubica en el grupo mexicanus (Soleglad, 1973; Sissom, 2000). Son animales de tamaño pequeño a mediano, con longitud total de 24.2 $\mathrm{mm}$ en el holotipo (intervalo $=23.30-29.40$ ) y longitud del cefalotórax de $3.30 \mathrm{~mm}($ rango=3.2-3.5). Mano (quela sin considerar el dedo fijo) de apariencia redondeada y corta, $1.52-2$ veces más larga que ancha $(p=0.347)$ y 1.81 a 1.94 más larga que alta $(p=0.527)$, independientemente del sexo; con el dedo móvil casi del mismo tamaño que la longitud de cefalotórax y la longitud de la mano (véase medidas completas en el Cuadro 1). Conteo de dientes pectinales en machos $12-13$ (50\% con 12 y $50 \%$ con 13$)$, mientras que para hembras es $11-12$ (90\% con 11). Carapacho con una pequeña muesca en la parte media del borde anterior(Fig. 2); dedo móvil de la quela del pedipalpo con fila principal de gránulos dividido en 6 subhileras por 5 dentículos de mayor tamaño, con 6 dentículos accesorios; dedo fijo con fila principal de gránulos dividido en 6 subhileras por 5 dentículos de mayor tamaño, con 5 dentículos accesorios. Color base castaño oscuro; con manchas negruzcas en cefalotórax, quelíceros, pedipalpos, tergitos mesosomales y patas; tergito VII con área sin pigmentar en forma de $\mathrm{T}$ delgada (Fig. 3); esternito $\mathrm{V}$ de los machos con parche sin pigmentar en la parte media posterior de forma triangular ocupando 1/3 de la longitud del esternito (Fig. 4)

Holotipo, macho adulto. Coloración. Castaño obscuro, con manchas negruzcas en cefalotórax, quelíceros, pedipalpos tergitos mesosomales y patas. Borde posterior del carapacho 


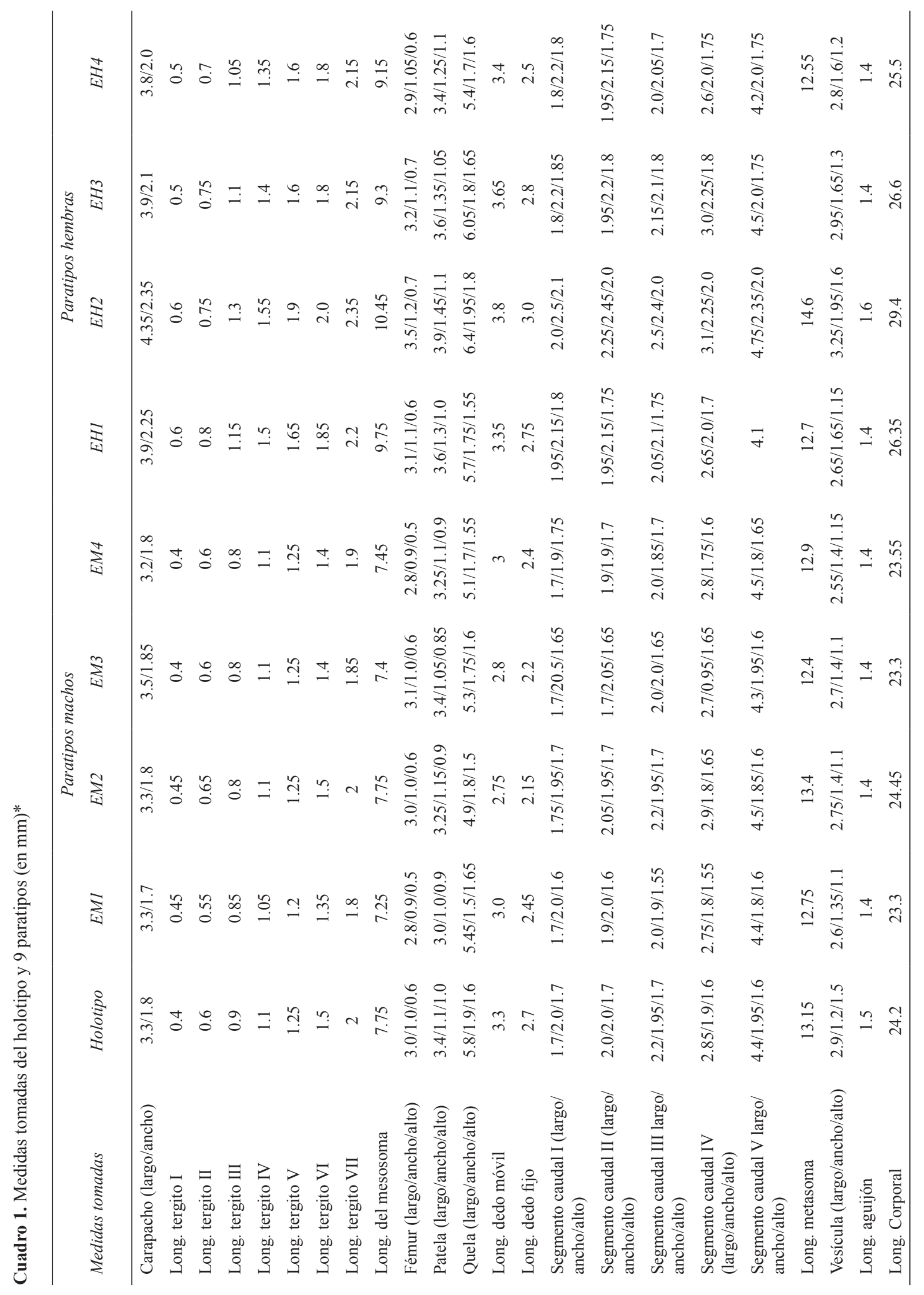



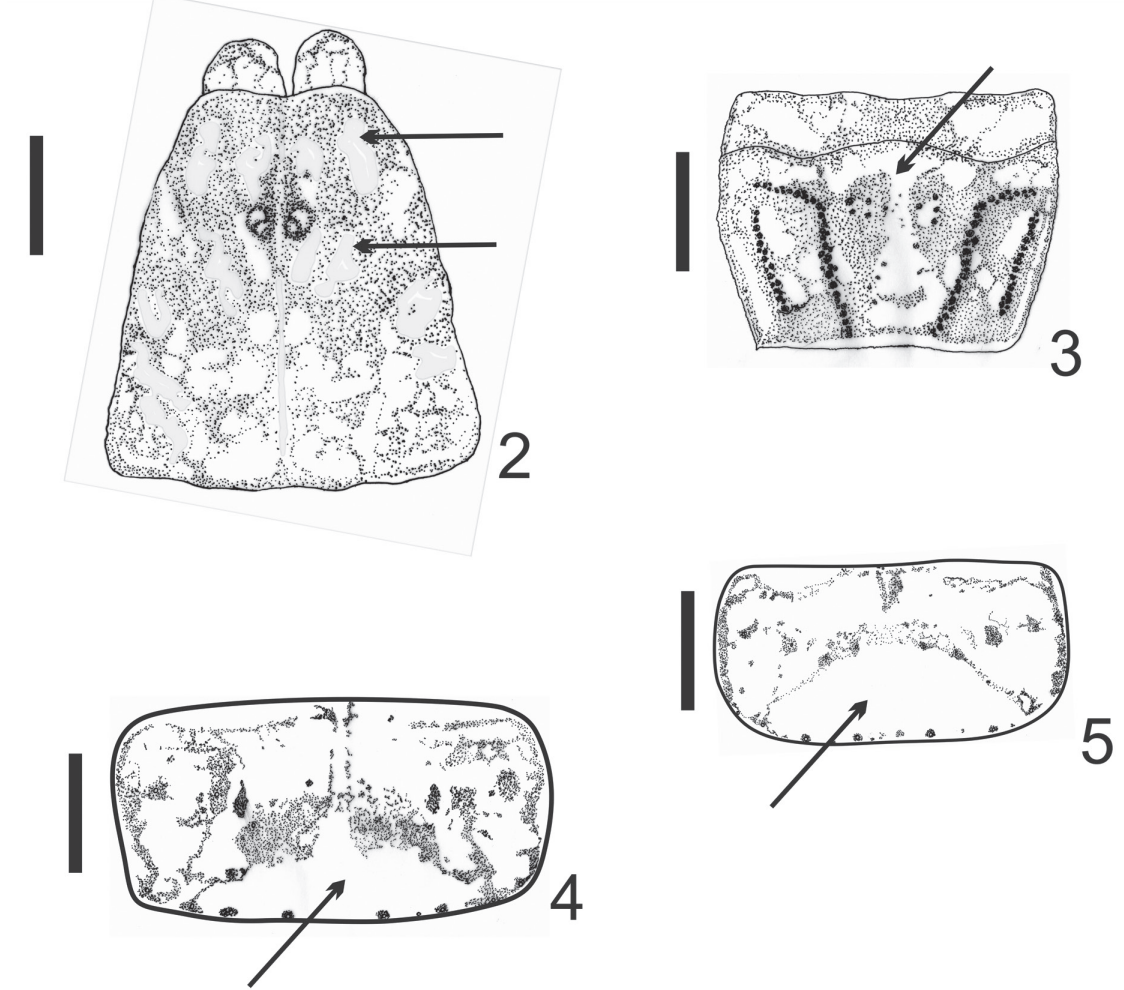

Figuras 2-5. Características morfológicas de Vaejovis morelia sp. nov., macho. 2, carapacho; 3, vista del tergito VII; 4, esternito V con parche triangular sin pigmentación; 5, vista del esternito V de Vaejovis pusillus con el parche redondeado sin pigmentación. Escala de la barra $1 \mathrm{~mm}$. Las flechas señalan características relevantes.

con pigmentación negruzca. Tubérculo ocular negro, con el pigmento proyectándose anteriormente sin alcanzar el borde del carapacho, posteriormente se continúa a lo largo del surco medio, dejando la parte posterior del mismo sin pigmento. Zona anterior a los ojos laterales unida por pigmento oscuro que cubre toda la superficie y se proyecta hacia atrás sobre los ojos laterales y a los lados del surco medio formando 4 áreas claras en la parte anterior de los ojos medios y otras 4 áreas claras por debajo de ellos (Fig. 2); lateralmente se definen 3 áreas claras de las cuales las posteriores son pequeñas y orientadas hacia el surco medio. Lóbulos presentan pigmentación en la parte media posterior sin alcanzar el margen, definiendo de 4 a 5 áreas pequeñas sin pigmento, 2 de ellas bien definidas en la parte posterior de cada lóbulo. Con mancha más o menos difusa y reticulada, que corre paralelamente al borde posterior del carapacho (Fig. 2). Dorsalmente, base de los quelíceros con pigmentación reticulada, más intensa en margen anterior proyectándose hacia base del dedo fijo; dedo móvil con más de la mitad de la superficie de color negro sin incluir los dientes; ventralmente, el pigmento ocupa $2 / 3$ partes del dedo móvil dejando sin pigmentación la parte distal. Mesosoma con pretergitos I-VI con pigmentación sobre la mayor parte de la superficie, con 2 áreas pequeñas semicirculares sin pigmento en la parte media; pretergito VII con pigmentación concentrada en parte media; postergitos I-VI con área mesal sin pigmentación y a cada lado con 2 manchas, las cuales forman 2 bandas obscuras, separadas por área sin pigmento, que recorren longitudinalmente el mesosoma; en parte anterior del tergito VII las bandas se aprecian unidas entre las carenas medias, dejando un área bien definida sin pigmentar con forma de T (Fig. 2). Prosoma y mesosoma en vista ventral con color base más claro que en el dorso y con pigmentación moderada en coxapófisis I y II, esternón, opérculo genital, base de los peines y esternitos. Dorsalmente, pigmentación de los segmentos metasomales I-III concentrada en parte media anterior, uniéndose con borde posterior con 2 líneas, segmento IV pigmentación en parte media uniforme sobre toda la longitud del segmento, segmento $\mathrm{V}$ sin pigmentación dorsal, lateral y ventralmente con pigmentación sobre las carenas y la superficie de la parte media y anterior. Vesícula ventralmente con pigmentación tenue sobre partes centrales y laterales, recorriendo todo el telson dejando 2 bandas de color claro en la zona medioventral, lateralmente con pigmentación tenue sobre su parte superior y dorsalmente sin pigmentación.

Carapacho. Borde anterior casi recto y liso, con ligera muesca en parte media (Fig. 2); con 6 setas, bordes 
laterales muy finamente crenulados con 1 seta a cada lado a la altura del último ojo lateral; borde posterior ligeramente granuloso. Superficie con granulación fina y densa, las áreas pigmentadas con gránulos de mayor tamaño, definiendo áreas sin pigmento, principalmente en parte media posterior; con par de setas medias laterales ubicadas sobre la mancha de mayor tamaño a la altura del borde posterior del tubérculo ocular.

Quelíceros. Sérrula bien definida y ocupando un poco menos de la mitad distal del dedo; con una seta en el área dorsoventral de la base del quelícero, ubicada en área clara que deja el pigmento entre la base del dedo fijo y el dorso de la base del quelícero.

Mesosoma en vista dorsal. Tergitos I-V con los bordes laterales finamente crenulados y el borde posterior granuloso, superficie con granulación fina, irregular y densa, con el área de la carena media finamente granulosa. Carena media ausente en tergitos I y II, en III-VII de vestigial a muy débil. En tergito VII las carenas submedias y laterales se observan fuertes y granulosas.

Prosoma y mesosoma en vista ventral. Superficie de coxapófisis I y II, esternón, opérculo genital y base de los peines setosos, con fina y densa granulación; papilas genitales bien desarrolladas; base de los peines con el borde anterior redondeado y un surco medio que recorre 3/4 la longitud de la base de los peines, borde posterior recurvado; con 12-13 dientes pectíneos; esternitos III, IV, V y VI con bordes laterales finamente crenulados y borde anterior liso, superficie en parte media lisa y hacia los bordes laterales con granulación fina; en esternito III se cuentan 21 setas, 5 en la mitad anterior, 6 en la mitad posterior y 10 sobre el borde posterior; en esternitos IV y V hay 16 setas, 8 ubicadas en la mitad anterior y 8 en el borde posterior; en el $\mathrm{V}$ presenta un parche sin pigmentar en la parte media posterior, el cual es triangular y ocupa $1 / 3$ de la longitud del esternito sin alcanzar todo el ancho de la placa, disminuyendo su grosor hacia el borde posterior dejando aproximadamente $1 / 3$ del ancho del esternito con pigmento distribuido a ambos lados del mismo (Fig. 5); en el esternito VI hay 14 setas, 8 ubicadas en la mitad anterior y 6 sobre el borde posterior; esternito VII con bordes laterales moderadamente crenulados; no hay quillas submedianas y laterales muy poco aparentes, presenta 12 setas, 6 ubicadas en la mitad anterior, 2 en la mitad posterior cerca de los bordes laterales y posteriores y 4 más sobre el borde posterior. Patas: tercer telotarso con un sólo par de espínulas distales.

Metasoma. Segmentos I, II, III con carenas dorsolaterales y laterales supramedianas fuertes y granulares, segmento I con carenas laterales inframedianas y ventrolaterales moderadas y granulares, carenas submedianas ventrales débiles y granulares, espacios intercarenales con ligera y densa granulación, áreas pigmentadas con gránulos gruesos y bien definidos. Segmento II con carenas laterales inframedianas cortas, recorriendo $2 / 3$ del segmento en dirección postero-anterior, moderadas $\mathrm{y}$ granulares, carenas ventrolaterales fuertes y granuladas, carenas submedianas ventrales moderadas y granulares, espacios intercarenales con ligera y densa granulación, áreas pigmentadas con gránulos gruesos y bien definidos. Segmento III con carenas laterales inframedianas más cortas que en el segmento II recorriendo aproximadamente $1 / 3$ del segmento en dirección postero-anterior, débiles y granulares, carenas ventrolaterales fuertes y granulares, carenas submedianas ventrales moderadas y granulares, espacios intercarenales con ligera y densa granulación, áreas pigmentadas con gránulos gruesos y bien definidos. Segmento IV con todas las carenas fuertes y granulares, excepto las laterales inframedianas que no están presentes, espacios intercarinales con ligera y densa granulación, áreas pigmentadas con gránulos gruesos y bien definidos. Segmento V (Figs. 6, 7) con las carenas dorsolaterales $\mathrm{y}$ medio laterales moderadas y granulares, carenas ventrolaterales y ventromediana fuertes y granulares, espacios intercarenales con ligera y densa granulación, áreas pigmentadas con gránulos gruesos y bien definidos. Setas de carenas de segmentos I-IV: dorsolateral 0/0 - 0/1 - 1/1 - 2/2, lateral supramediana $0 / 0$ - 1/2 - 2/2 - 2/2, lateral

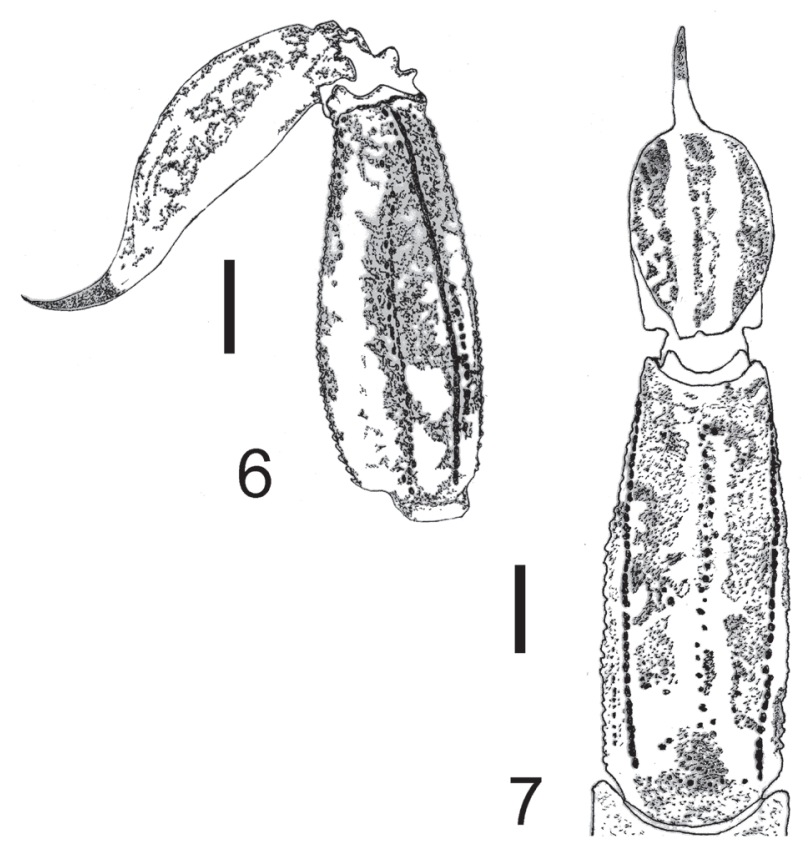

Figuras 6-7. Características morfológicas de Vaejovis morelia sp. nov., macho. 6, vista lateral del segmento caudal V y vesícula; 7 , vista ventral segmento caudal V y vesícula. Escala de la barra $1 \mathrm{~mm}$. 
inframediana $1 / 1-0 / 0-0 / 0-0 / 0$, ventrolateral $2 / 2-2 / 3$ - $3 / 3$ - 3/3, submediana ventral $2 / 2-3 / 3-3 / 3-3 / 3$; en segmento V: dorsolateral $4 / 4$ mediolateral $3 / 3$, ventrolateral 5/5. Vesícula: Setosa, lateralmente con aspecto alargado, ventralmente con aspecto ovalado (Figs. 6, 7), tubérculo subaculear débil, superficie granulosa.

Pedipalpo. Ortobotriotaxia tipo C; trocánter con 8 setas, 3 ubicadas ventralmente, 3 dorsalmente, 2 en cara interna; fila de gránulos gruesos bien definidos que le recorren longitudinalmente, superficie con granulación fina y densa intercalada con gránulos gruesos bien definidos. Fémur. Carena prodorsal moderada a fuerte y granular con 1 seta ubicada en la parte media; carena retrodorsal moderada y granular en el área proximal y ligeramente granulosa en el área distal, con 2 setas, 1 en la parte media y la otra entre la área distal y el área media (Fig. 8); carena proventral moderada a fuerte y granular, sin setas; carena retroventral débil a vestigial y con gránulos bien definidos pero dispersos, con 3 setas distribuidas uniformemente sobre la carena; superficie dorsal con granulación densa y fina intercalada con gránulos gruesos bien definidos; superficie interna con granulación densa y fina; cara interna hacia la mitad proximal con gránulos gruesos y bien definidos, con 7 setas, 5 ubicadas hacia la mitad proximal y 2 más junto al margen distal; superficie ventral con granulación fina y densa, con 1 fila de gránulos gruesos bien definidos concentrados principalmente en la región proximal y disminuyendo hacia la distal. Patela. Carena prodorsal fuerte y granular, carenas retrodorsal y proventral moderadas y granulares, carena retroventral moderada a fuerte y granular, carena externa débil y granular; superficie dorsal con granulación densa y fina (Figs. 9, 10), superficie interna con granulación densa y fina, superficie ventral con granulación fina y densa con 1 fila de gránulos gruesos bien definidos concentrados principalmente en la parte proximal y disminuyendo hacia la parte distal, superficie externa con granulación fina y densa, con 5 setas ubicadas en la parte media, 1 en la mitad distal y 2 cerca del borde distal. Quela. Con apariencia robusta, carena digital bien desarrollada y granulosa, carena dorsal secundaria bien desarrollada y granulosa, carena externa secundaria bien desarrollada y lisa, carena dorsomarginal bien desarrollada y granulosa, carenas mediainterna y ventrointerna débiles y granulosas, carena dorsointerna bien desarrollada y granulosa. Superficie de la quela con granulación fina y densa (Figs. 11, 12). Dedo fijo con hilera de dentículos dividida en 6 subhileras por 5 dentículos más grandes, hilera basal más larga y con 5 dentículos accesorios en la cara interna. Dedo móvil con hilera de dentículos dividida en 6 subhileras por 5 dentículos más grandes, hilera basal más larga, 6 dentículos accesorios en la cara interna. Tricobotrias $i b$-it ubicadas en la parte basal del dedo fijo, no en la palma de la mano (Fig. 13).
Hemiespermatóforo. Estructura poco compleja, con laminilla uniforme, ganchos basales cortos, lámina recta y angosta, poco esclerotizada (Fig. 14). No hay tapón copulatorio.

Dimorfismo. Las hembras son estadísticamente de mayor longitud corporal $(p<0.05)$ (Cuadro 1), conteo de dientes pectinales en machos $12-13$ ( $50 \%$ con 12 y $50 \%$ con 13$)$, mientras que para hembras es de $11-12(90 \%$ con 11$)$. En machos se presenta un parche sin pigmentación en el esternito $\mathrm{V}$, que en las hembras no existe. Estadísticamente $(p<0.05)$, la vesícula en los machos es menos ancha (1.41 $\mathrm{mm} \pm 0.5)$ que la de las hembras $(1.69 \mathrm{~mm} \pm 0.14)$. Patela más ancha en hembras $(1.33 \mathrm{~mm} \pm 0.07)$ que en machos $(1.08 \mathrm{~mm} \pm 0.05)$, mientras que el dedo fijo en los machos es estadísticamente más corto $(2.38 \mathrm{~mm} \pm 0.21)$ que el de las hembras $(2.78 \mathrm{~mm} \pm 0.18)$. Proporción longitud mano/ longitud dedo móvil también mostró diferencia estadística con valor promedio de $0.98 \pm 0.07$ para machos y $0.88 \pm$ 0.008 para hembras, caracterizándose los machos por tener quelas con dedo del mismo tamaño que la mano, mientras que en las hembras la mano es más pequeña.

Variación. Fila de gránulos del trocánter que lo recorren longitudinalmente de muy débiles a gruesos y bien definidos; variación de las setas metasomales se muestra en los cuadros 2 y 3 . Setas del esternito III de 18 a 21 en machos y en hembras de 18 a 26.

Resumen taxonómico

Material tipo. México: estado de Michoacán: Holotipo CNAN-T0081 y 3 paratipos (2 hembras y 1 macho adultos) CNAN-T0082; 2 paratipos (macho y hembra), depositados en el American Museum of Natural History de Nueva York, (AMNH); 2 paratipos (macho y hembra) que quedaron depositados en el Instituto Nacional de Referencia Epidemiológica, México (INDRE) y 2 paratipos (macho y hembra) para la Colección Aracnológica de la Facultad de Biología de la Universidad Michoacana de San Nicolás de Hidalgo (CAFBUM). Todos los ejemplares fueron colectados el 10 de agosto de 2010 por M. Villaseñor, V. Guzmán y E. Miranda, entre la hojarasca y troncos caídos del bosque Lázaro Cárdenas, en la ciudad de Morelia, Michoacán, México. Coordenadas 1940'46" N, 101 ${ }^{\circ} 10^{\prime} 39^{\prime \prime} \mathrm{O}$ a 2030 m snm (Fig.1).

Distribución. Esta especie es conocida de la localidad tipo y de la localidad cercana, nombrada Los Filtros Viejos del municipio de Morelia, Michoacán (Fig. 1).

Etimología. El nombre de la especie se refiere a Morelia, ciudad donde se forjó la independencia de México. El nombre se usa como sustantivo en aposición.

Comentarios taxonómicos

Vaejovis morelia sp. nov. es similar a $V$. pusillus y $V$. dzahui. Se distingue de V. pusillus por las siguientes características: 1) en promedio es de mayor tamaño (sin incluir aguijón), machos de longitud corporal total de 

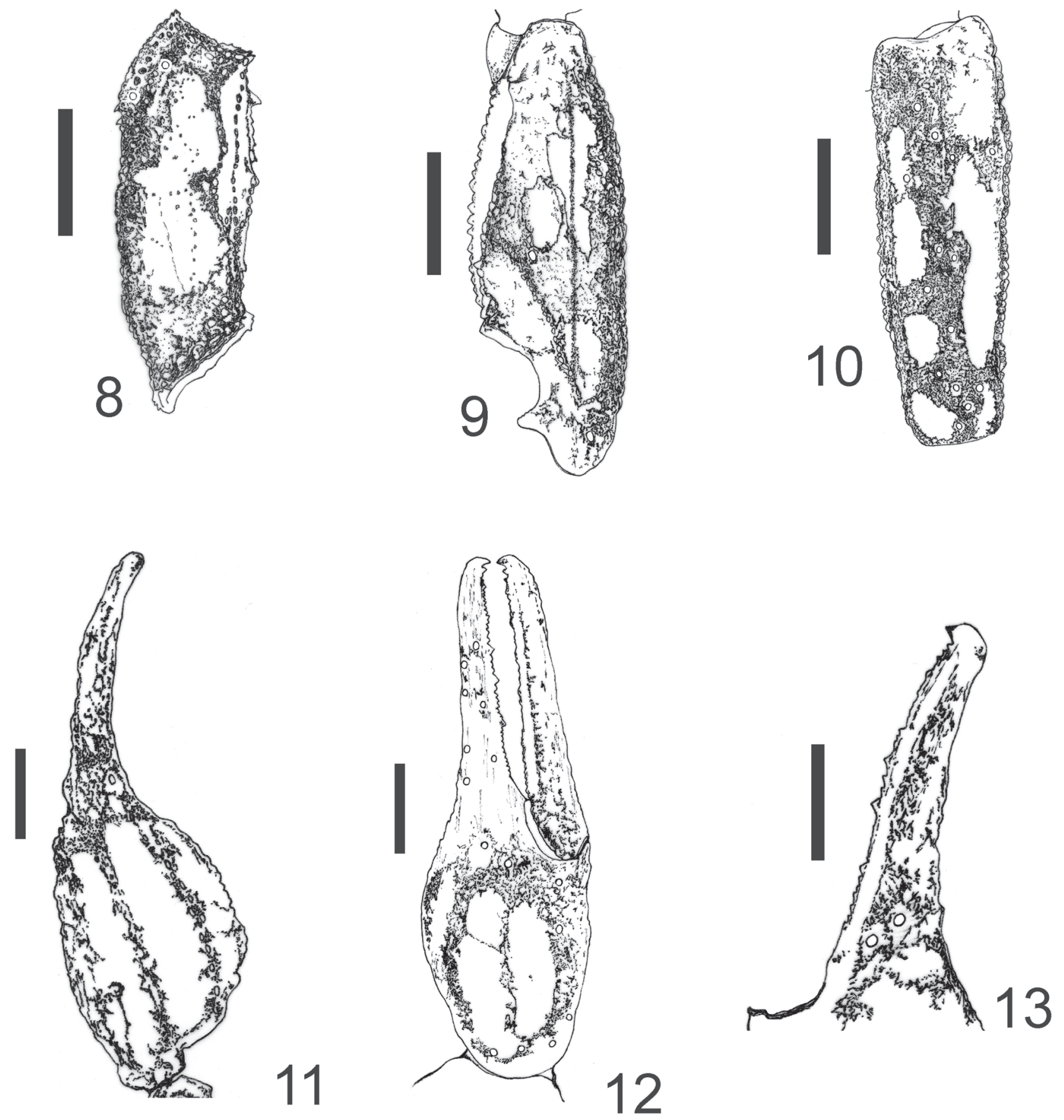

Figuras 8-13. Características morfológicas de Vaejovis morelia sp. nov., macho. 8, vista dorsal fémur; 9, vista dorsal patela; 10, Vista retrodorsal patela; 11, vista dorsal quela; 12, vista retrodorsal de la quela; 13, vista interna del dedo fijo. Escala de la barra $1 \mathrm{~mm}$. 

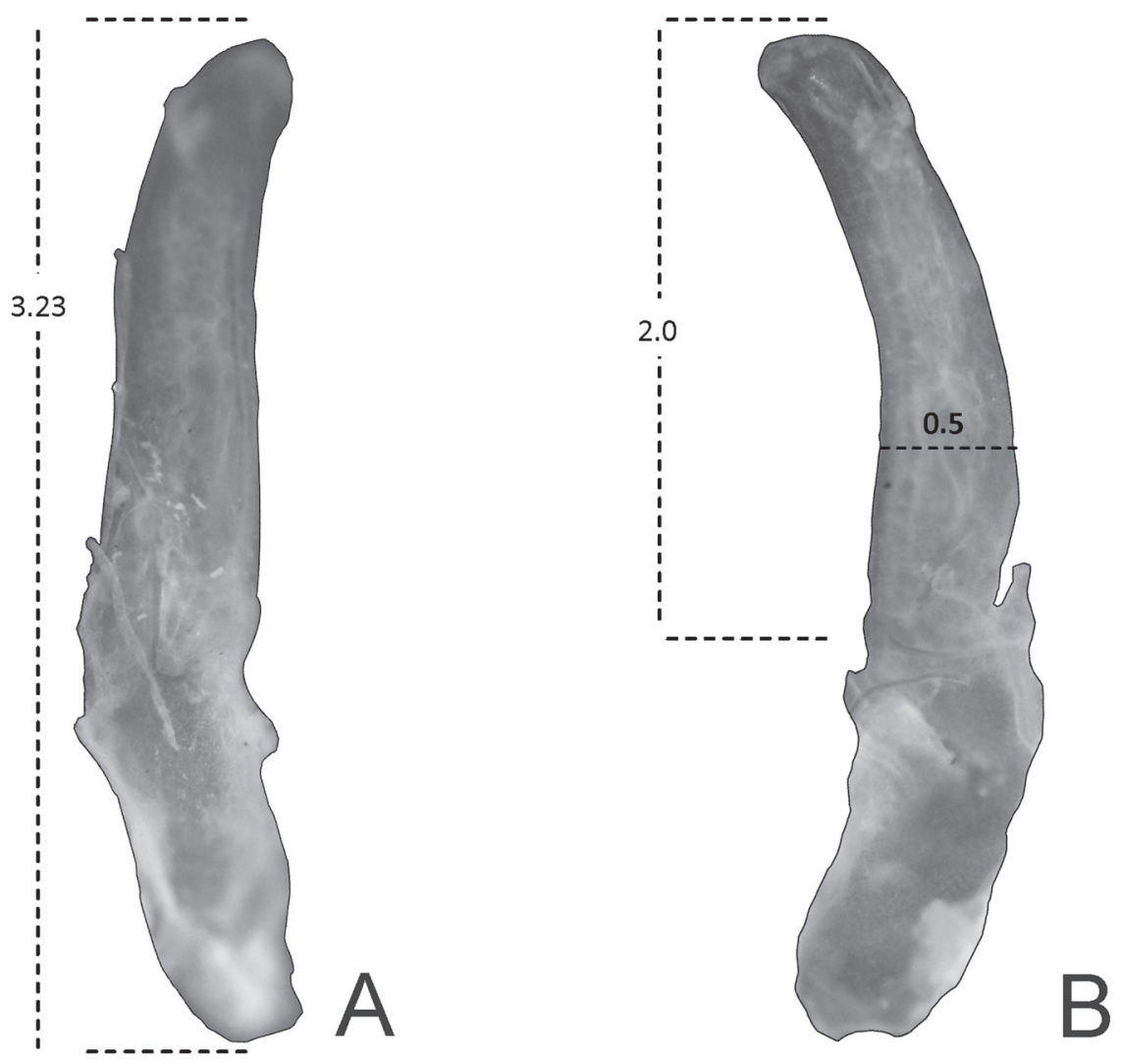

Figura 14. Morfología del hemispermatóforo de Vaejovis morelia sp. nov. A, vista dorsal y B, vista ventral.

Cuadro 2. Conteo de las setas de las carenas metasomales de los segmentos I-IV. La frecuencia se muestra entre paréntesis

\begin{tabular}{|c|c|c|c|c|c|c|c|c|c|c|}
\hline \multicolumn{11}{|c|}{ Segmentos metasomales } \\
\hline \multirow{2}{*}{ Carenas metasomales } & \multicolumn{2}{|c|}{$I$} & \multicolumn{2}{|c|}{ II } & \multicolumn{2}{|c|}{ III } & \multicolumn{2}{|c|}{$I V$} & \multicolumn{2}{|c|}{$V$} \\
\hline & Macho & Hembra & Macho & Hembra & Macho & Hembra & Macho & Hembra & Macho & Hembra \\
\hline \multirow[t]{3}{*}{ Dorsolateral } & $0 / 0(3)$ & $0 / 0(2)$ & $0 / 1(1)$ & $1 / 0(2)$ & $1 / 1(5)$ & $1 / 1(5)$ & $2 / 2(5)$ & $1 / 2(1)$ & $4 / 4(4)$ & $4 / 4(5)$ \\
\hline & $1 / 0(1)$ & $1 / 0(2)$ & $1 / 1(4)$ & 1/1 (3) & & & & $2 / 2(4)$ & $5 / 5(1)$ & \\
\hline & $1 / 1(1)$ & $1 / 1(1)$ & & & & & & & & \\
\hline \multirow[t]{3}{*}{ Lateral supramediana } & $0 / 0(4)$ & $0 / 0(4)$ & $1 / 2(1)$ & $1 / 1(5)$ & $1 / 1(3)$ & $1 / 1(4)$ & $2 / 2(5)$ & $2 / 2(5)$ & - & - \\
\hline & 0/1 (1) & $1 / 0(1)$ & $1 / 1(4)$ & & 2/1 (1) & $2 / 3(1)$ & & & & \\
\hline & & & & & $2 / 2(1)$ & & & & & \\
\hline \multirow[t]{2}{*}{ Lateral inframediana } & $1 / 1(4)$ & $1 / 1(5)$ & $0 / 0(5)$ & $0 / 0(4)$ & $0 / 0(5)$ & $0 / 0(5)$ & $0 / 0(5)$ & $0 / 0(5)$ & - & - \\
\hline & $2 / 2(1)$ & & & 0/1 (1) & & & & & & \\
\hline \multirow[t]{3}{*}{ Ventrolateral } & $2 / 2(5)$ & $2 / 2(4)$ & $2 / 2(3)$ & $2 / 2(1)$ & $2 / 2(2)$ & $3 / 3(5)$ & $2 / 2(1)$ & $3 / 3(5)$ & $4 / 3(1)$ & $4 / 4(5)$ \\
\hline & & 2/1 (1) & $2 / 3(1)$ & $3 / 3(4)$ & $3 / 3(3)$ & & $3 / 3(4)$ & & $4 / 4(3)$ & \\
\hline & & & $3 / 3(1)$ & & & & & & $5 / 5(1)$ & \\
\hline
\end{tabular}


Cuadro 2. Continúa

\begin{tabular}{|c|c|c|c|c|c|c|c|c|c|c|}
\hline \multirow{3}{*}{ Carenas metasomales } & \multicolumn{8}{|c|}{ Segmentos metasomales } & & \\
\hline & \multicolumn{2}{|c|}{$I$} & \multicolumn{2}{|c|}{$I I$} & \multicolumn{2}{|c|}{$I I I$} & \multicolumn{2}{|c|}{$I V$} & \multicolumn{2}{|c|}{$V$} \\
\hline & Macho & Hembra & Macho & Hembra & Macho & Hembra & Macho & Hembra & Macho & Hembra \\
\hline Ventral submediana & $2 / 2(5)$ & $\begin{array}{l}2 / 2(4) \\
2 / 3(1)\end{array}$ & $3 / 3(5)$ & $3 / 3(5)$ & $3 / 3(5)$ & $3 / 3(5)$ & $3 / 3(5)$ & $3 / 3(5)$ & - & - \\
\hline Media Lateral & - & - & - & - & - & - & - & - & $\begin{array}{l}2 / 2(2) \\
3 / 3(3)\end{array}$ & $3 / 3(5)$ \\
\hline
\end{tabular}

$23.76 \mathrm{~mm}( \pm 0.53)$ y $26.87 \mathrm{~mm}( \pm 1.48)$ en hembras, mientras que en $V$. pusillus los machos miden $16.62 \mathrm{~mm}$ $( \pm 0.76)$ y las hembras $20.61 \mathrm{~mm}( \pm 1.15)$; 2) quela más robusta en $V$. morelia sp. nov. con la proporción longitud/ ancho en machos de 2.72 a $3.63(n=5)$ y de 3.18 a 3.47 en hembras $(\mathrm{n}=5)$, mientras que en $V$. pusillus es de 3.29 a 3.72 en machos $(n=5)$ y en hembras de 3.64 a 4.25 $(n=4)$; 3) las quillas de la quela, externa secundaria, digital y dorso marginal están bien desarrolladas en $V$. morelia sp. nov., mientras que en $V$. pusillus son débiles y 4) en $V$. morelia sp. nov. el parche del esternito $\mathrm{V}$ en machos adultos es de forma triangular ocupando $1 / 3$ de la longitud del esternito, mientras que en $V$. pusillus el parche es de forma semicircular y ocupa más de la mitad de la longitud del esternito (Figs. 4, 5).

Vaejovis dzahui se distingue principalmente por la ausencia de un parche ventral en los machos; además, en la mano sólo hay 3 carenas evidentes, débiles a vestigiales (sensu Santibañez-López y Francke 2010). Adicionalmente, puede apreciarse que $V$. morelia sp. nov. es en promedio de mayor tamaño (machos $23.76 \mathrm{~mm}$ de longitud total $[ \pm 0.53] \mathrm{y}$ hembras de $26.87 \mathrm{~mm}$ [ \pm 1.48$])$; en $V$. dzahui los machos miden $15.76 \mathrm{~mm}( \pm 0.97)$ y las hembras $18.21 \mathrm{~mm}( \pm 1.17)$ de longitud total.

\section{Agradecimientos}

Al Consejo Nacional de Ciencia y Tecnología (CONACYT), por la beca 239256 otorgada a E. Pabel Miranda-López para los estudios de maestría. A la Coordinación de la Investigación Científica (CIC) de la Universidad Michoacana de San Nicolás de Hidalgo (UMSNH) y al proyecto Código de Barras para Arácnidos de México, financiado por CONACyT por apoyar el trabajo de campo. A Marco A. Villaseñor y Víctor M. Guzmán Pérez por su ayuda en el trabajo de campo. A Paola Miranda por su ayuda en la realización de los dibujos y a Carlos Santibañez del IBUNAM, por sus comentarios y ayuda en la toma de fotografías. Los ejemplares estudiados se colectaron bajo el amparo de la licencia de colector científico FAUT-0175 otorgada por la Secretaría del Medio Ambiente y Recursos Naturales (SEMARNAT) a O. F. Francke.

\section{Literatura citada}

Acosta, L. E., D. M. Candido, E. H. Buckup y A. D. Brescovit. 2008. Description of Zabius gaucho (Scorpiones, Buthidae), a new species from southern Brazil, with an update about the generic diagnosis. Journal of Arachnology 36:491-501.

Atlas Geográfico del Estado de Michoacán. 2003. Secretaría de Educación del Estado de Michoacán/ Universidad Michoacana de San Nicolás de Hidalgo/ EDDISA, Morelia, Michoacán. p. 42-46.

Ayrey, R. F. y M. E. Soleglad. 2011. A new species of Vaejovis from Prescott, Arizona (Scorpiones: Vaejovidae). Euscorpius 114:1-15.

Francke, O. F. 1977. Scorpions of the genus Diplocentrus from Oaxaca, México. Journal of Arachnology 4:145-200.

Graham, M. 2007. Sky Island Vaejovis: Two new species and a redescription of $V$. vorhiesi Stahnke (Scorpiones: Vaejovidae). Euscorpius 51:1-16.

Graham, M y R. W. Bryson. 2010. Vaejovis montanus (Scorpiones: Vaejovidae), a new species from the Sierra Madre Occidental of Mexico. Journal of Arachnology 38:285-293.

Hendrixson, B. y W. D. Sissom. 2001. Descriptions of two new species of Vaejovis C.L. Koch, 1836 from Mexico, with a redescription of Vaejovis pusillus Pocock, 1898 (Scorpiones: Vaejovidae). In Scorpions 2001. In memoriam Gary A. Polis, V. Fet y P. A. Selden. (eds.). British Arachnological Society, Burnham Beeches, Buckinghamshire. p. 215-223

Miranda-López, E., J. Ponce-Saavedra y O. F. Francke. 2010. Situación taxonómica y distribución actual de Vaejovis pusillus Pocock (Scorpiones:Vaejovidae). Ciencia Nicolaita 52:56-62.

Prendini, L. y W. Wheeler. 2005. Scorpion higher phylogeny and classification, taxonomic anarchy, and standards for peer review in online publishing. Cladistics 21:446-494.

Santibáñez-López, C. E. y O. F. Francke. 2010. New and poorly known species of the mexicanus group of the genus Vaejovis (Scorpiones: Vaejovidae) from Oaxaca, Mexico. Journal of Arachnology 38:555-571. 
Santibáñez-López, C. E. y W. D Sissom. 2010. A new species of the Vaejovis eusthenura group in Oaxaca, Mexico (Scorpiones: Vaejovidae). Zootaxa 2493:49-58.

Sissom, W. D. 2000. Family VaejovidaeThorell 1876. In Scorpions of the world (1758-1998), V. Fet, W. D. Sissom, G. Lowe y M. Braunwalder (eds.). Catalog of the New York Entomological Society. p. 503-553.

Sissom, W. D. 2011. A new species of the genus Vaejovis from Southwestern New Mexico (Arachnida: Scorpiones: Vaejovidae). Southwestern Entomologist 36:85-90.

Sissom, W. D., G. A. Polis y D. D. Watt. 1990. Field and laboratory methods. In The biology of scorpions, G. A. Polis (ed.). Stanford University Press. Stanford, California. p. 445-461.

Soleglad, M. E. 1973. Scorpions of the mexicanus group of the genus Vaejovis (Scorpionida, Vejovidae). Wasmann Journal of Biology 31:351-372.
Soleglad, M. E. y V. Fet. 2008. Contributions to scorpion systematics. III. Subfamilies Smeringurinae and Syntropinae (Scorpiones: Vaejovidae). Euscorpius 71:1-115.

Stahnke, H. L. 1970. Scorpion nomenclature and mensuration. Entomological News 81:297-316.

Stahnke, H. L. 1972. U.V. light, a useful field tool. BioScience 22:604-607.

Vachon, M. 1974. Étude des caractères utilisés pour classer les familles et les genres de Scorpions (Arachnides). 1. La trichobothriotaxie en Arachnologie, Sigles trichobothriaux et types de trichobothriotaxie chez les Scorpions. Bulletin du Muséum National d'Histoire Naturelle, Paris, 140 (Zool. 104), mai-juin 1973:857-958.

Zárate-Gálvez, K. y O. F. Francke. 2009. Nueva especie de Vaejovis (Scorpiones: Vaejovidae) de Chiapas, México. Revista Ibérica de Aracnología 17:21-28. 